Nonlinear Analysis: Theory, Methods and Applications, 71, (2009),e186-e190. doi:10.1016/j.na.2008.10.011

*Email: ramm@math.ksu.edu 


\title{
Preparing materials with a desired refraction coefficient
}

\author{
A. G. Ramm†* \\ $\dagger$ Mathematics Department, Kansas State University, \\ Manhattan, KS 66506-2602, USA
}

\begin{abstract}
A recipe is given for creating material with a desired refraction coefficient by embedding many small particles in a given material. To implement this recipe practically, some technological problems have to be solved. These problems are formulated.
\end{abstract}

PACS 43.20.+g, 62.40.+d, 78.20.-e.

MSC 35J10, 74J25, 81U40

Keywords: metamaterials; nanotechnology; refraction coefficient; negative refraction, wave focusing

\section{Introduction}

In a series of papers [5]-[13] and in the monographs [4], [3], the author has developed a wave scattering theory by many small bodies of arbitrary shapes and, on this basis, proposed a method for creating materials with some desired properties. The goal of this paper is to explain this method and to formulate two nanotechnological engineering problems that should be solved in order for the proposed method can be implemented practically.

Let us formulate the statement of the problem. Let $D \subset \mathbb{R}^{3}$ be a bounded domain filled in with a material with a known refraction coefficient. The scalar wave scattering problem consists of finding the solution to the Helmholtz equation:

$$
\begin{gathered}
L_{0} u:=\left(\nabla^{2}+k^{2} n_{0}^{2}(x)\right) u=0 \quad \text { in } \quad \mathbb{R}^{3}, \quad \operatorname{Im} n_{0}^{2}(x) \geq 0, \\
u=u_{0}+v, \quad u_{0}:=e^{i k \alpha \cdot x}, \\
v=A_{0}(\beta, \alpha) \frac{e^{i k r}}{r}+o\left(\frac{1}{r}\right), \quad r:=|x| \rightarrow \infty, \quad \beta:=\frac{x}{r} .
\end{gathered}
$$

The function $n_{0}(x)$ is assumed bounded, piecewise - continuous,

$$
n(x)=1 \quad \text { in } \quad D^{\prime}:=\mathbb{R}^{3} \backslash D, \quad \operatorname{Im} n_{0}^{2}(x) \geq 0 .
$$

The function $A(\beta, \alpha)$ is called the scattering amplitude. The wavenumber $k=\frac{2 \pi}{\lambda}$, where $\lambda$ is the wavelength in $D^{\prime}, \alpha \in S^{2}$ is the direction of the incident plane wave $u_{0}, S^{2}$ is the 
unit sphere in $\mathbb{R}^{3}, \beta \in S^{2}$ is the direction of the scattered wave. The solution to problem (1)-(3) is called the scattering solution. It is well-known ([2]) that this solution exists and is unique under our assumptions, namely $n_{0}^{2}(x)$ is bounded $\sup _{x \in \mathbb{R}^{3}}\left|n_{0}^{2}(x)\right| \leq n_{0}=$ const, $\operatorname{Im} n_{0}^{2}(x) \geq 0, n_{0}^{2}(x)=1$ in $D^{\prime}$, so that $A_{0}(\beta, \alpha)$ is determined uniquely if $n_{0}^{2}(x)$ is given. We assume $k>0$ fixed and do not show the dependence of $A_{0}(\beta, \alpha)$ on $k$. The operator $L_{0}$ at a fixed $k>0$ can be considered as a Schroedinger operator $L_{0}=\nabla^{2}+k^{2}-q_{0}(x)$, where $q_{0}(x):=k^{2} n_{0}^{2}(x)-k^{2}$.

Problem(P): We want to construct a material in $D$ with a desired refraction coeffcient, i.e., with a desired function $n^{2}(x), \operatorname{Im} n^{2}(x) \geq 0$.

Why is this problem of practical interest? We give just two reasons (out of, possibly, many). First, creating a refraction coefficient such that the corresponding material has negative refraction is of practical interest. One says that a material has negative refraction if the group velocity in this material is directed opposite to the phase velocity.

Secondly, creating such a refraction coefficient that the corresponding scattering amplitude $A(\beta)=A(\beta, \alpha)$ at a fixed $\alpha \in S^{2}$ (and a fixed $k>0$ ) approximates with any desired accuracy $\epsilon>0$ an arbitrary given function $f(\beta) \in L^{2}\left(S^{2}\right)$ is of practical interest. This problem we call the problem of creating material with a desired wave-focusing property.

In Section 2 we explain how to create such a material. In Section 3 we discuss the technological aspects of our recipe for creating material with a desired refraction coefficient. Creating material with negative refraction is discussed in Section 4.

\section{Creating material with a desired refraction coefficient}

The basic idea of our method is to embed in $D$ many small particles $D_{m}, 1 \leq m \leq M$, with a prescribed boundary impedance. The number of the embedded particles in an arbitrary open subdomain $\Delta \subset D$ is given asymptotically as

$$
\mathcal{N}(\Delta)=\frac{1}{\phi(a)} \int_{\Delta} N(x) d x[1+o(1)], \quad a \rightarrow 0 .
$$

Here $\phi>0$ if $a>0, \phi(0)=0, \phi$ is strictly monotonically growing, $N(x) \geq 0$ is a piecewise-continuous function which we can choose as we want. The smallness of the embedded particles means that $k a \ll 1$, where $a=\frac{1}{2} \max _{m} \operatorname{diam} D_{m}$, where $D_{m}$ is the $m$-th small particle. Let $M=M(a)$ be the total number of the embedded particles. For simplicity we assume that $D_{m}$ is a ball, centered at a point $x_{m} \in D$, of radius $a$. The distance $d:=\min _{m \neq j} \operatorname{dist}\left(D_{m}, D_{j}\right)$ between neighboring particles is much larger than $a$, $d \gg a$, but $d$ is not assumed much larger than $\lambda$, so in our theory there can be many small particles on the wavelength in $D$. This means that the distribution of the embedded particles is not necessarily "diluted".

The scattering problem in the region with embedded particles is formulated as follows:

$$
\begin{gathered}
L_{0} U=0 \quad \text { in } \quad \mathbb{R}^{3} \backslash \bigcup_{m=1}^{M} D_{m}, \\
U_{N}=\zeta_{m} U \quad \text { on } \quad S_{m}, \quad 1 \leq m \leq M,
\end{gathered}
$$




$$
U(x)=u(x)+V, \quad \frac{\partial V}{\partial r}-i k V=o\left(\frac{1}{r}\right), \quad r \rightarrow \infty .
$$

Here $N$ is the unit normal to $S_{m}$, pointing out of $D_{m}, S_{m}$ is the surface of $D_{m}, M$ is the total number of the embedded particles $D_{m}, \zeta_{m}$ is the boundary impedance, $\operatorname{Im} \zeta_{m} \leq 0$, $u$ is the scattering solution in the absence of the embedded particles, i.e., the solution to problem (1)-(3).

Let us assume that

$$
\zeta_{m}=\frac{h\left(x_{m}\right)}{a^{\varkappa}}, \quad-1<\varkappa<2, \quad \varkappa=\text { const }
$$

where $h(x)$ is a piecewise-continuous function in $D$,

$$
\operatorname{Im} h(x) \leq 0 .
$$

The function $N(x) \geq 0$ in (4) and $h(x), \operatorname{Im} h \leq 0$, we can choose as we wish. These functions will define the desired refraction coefficient of the new material which is obtained in the limit $a \rightarrow 0$. We assume that

$$
M=M(a)=O\left(a^{\varkappa-2}\right), \quad d=O\left(a^{\frac{2-\varkappa}{3}}\right),
$$

and the function $\phi(a)$ in $(5)$ is

$$
\phi(a)=a^{2-\varkappa} .
$$

The solution to problem (6)-(8) depends on $a, U=U(x, a)$, but we do not show this dependence for brevity. We also do not show the dependence of $U$ on $\alpha$ and $k$, because they are fixed. Our standing assumptions are (4), (5), (9)-(12). They are not repeated in the statements of theorems.

Denote by $g(x, y)$ the Green's function of the operator $L_{0}$ in the absence of the embedded particles, $L_{0} g(x, y)=-\delta(x-y)$ in $R^{3} ; g$ satisfies the radiation condition.

Theorem 1 Problem (6)-(8) has a solution, this solution is unique, and it is of the form:

$$
U(x)=u(x)+\sum_{m=1}^{M} \int_{S_{m}} g(x, s) \sigma_{m}(s) d s
$$

where the functions $\sigma_{m}$ are uniquely determined by the data, i.e., by $u_{0}(x, \alpha), \zeta_{m}, D_{m}$, $1 \leq m \leq M$, and $n_{0}^{2}(x)$.

Theorem 2 The solution $U$ to problem (6)-(8) has the following asymptotic as a $\rightarrow 0$

$$
U(x)=u(x)+\sum_{m=1}^{M(a)} g\left(x, x_{m}\right) Q_{m}+o(1), \quad a \rightarrow 0, \quad \inf _{m}\left|x-x_{m}\right| \geq d,
$$


where

$$
\begin{gathered}
Q_{m}:=\int_{S_{m}} \sigma_{m}(s) d s=-4 \pi h\left(x_{m}\right) u_{e}\left(x_{m}\right) a^{2-\varkappa}[1+o(1)], \quad a \rightarrow 0, \\
\sigma_{m}(s)=-h\left(x_{m}\right) u_{e}\left(x_{m}\right) a^{-\varkappa}[1+o(1)], \quad a \rightarrow 0,
\end{gathered}
$$

and

$$
u_{e}\left(x_{m}\right):=u_{e}^{(m)}\left(x_{m}\right), \quad u_{e}^{(m)}(x):=u(x)+\sum_{m^{\prime} \neq m} \int_{S_{m^{\prime}}} g(x, s) \sigma_{m^{\prime}}(s) d s
$$

Theorem 3 Assume that $f$ is an arbitrary bounded piecewise continuous function in $D$, and the points $x_{m}$ are distributed in $D$ so that (5) holds. Then

$$
\lim _{a \rightarrow 0} \phi(a) \sum_{m=1}^{M(a)} f\left(x_{m}\right)=\int_{D} f(x) N(x) d x
$$

Theorem 4 Under the assumptions (4), (5), (9)-(12) the following limit exists:

$$
\lim _{a \rightarrow 0} u_{e}^{(m)}(x)=u_{e}(x), \quad \forall x \in D .
$$

This limit does not depend on $m$ and is the unique solution to the equation

$$
u_{e}(x)=u(x)-\int_{D} g(x, y) p(y) u_{e}(y) d y
$$

where

$$
p(x)=4 \pi N(x) h(x) .
$$

Theorem 5 The limiting material in D, which is obtained as $a \rightarrow 0$, is described by the equation

$$
\begin{gathered}
L u_{e}=0 \quad \text { in } \quad D, \quad L:=\nabla^{2}+k^{2} n^{2}(x):=\nabla^{2}+k^{2}-q(x), \\
n^{2}(x):=n_{0}^{2}(x)-k^{-2} p(x), \quad p(x):=4 \pi N(x) h(x), \quad q(x):=q_{0}(x)+p(x), \\
u_{e}=u(x)+v_{e}(x),
\end{gathered}
$$

where $v_{e}(x):=-\int_{D} g(x, y) p(y) u_{e}(y) d y$.

The scattering amplitude, corresponding to $n^{2}(x)$, (or which is equivalent to the potential $q(x))$ is

$$
A(\beta, \alpha)=A_{0}(\beta, \alpha)-\frac{1}{4 \pi} \int_{D} u(y,-\beta) p(y) u_{e}(y) d y,
$$

where $u(x,-\beta)$ is the solution to problem (1)-(3) with $u_{0}=e^{-i k \beta \cdot x}$, that is, the scattering solution in the absence of the embedded particles when the incident direction of the plane wave $u_{0}$ equals $-\beta$. 
Remark 1 Formula (25) is based on Ramm's lemma (see [2, p. 46]) which says

$$
g(x, y)=\frac{e^{i k|y|}}{4 \pi|y|} u(x,-\beta)+O\left(\frac{1}{|y|^{2}}\right), \quad|y| \rightarrow \infty, \quad \beta:=\frac{y}{|y|},
$$

where $u(x,-\beta)$ is the scattering solution with the incident direction $-\beta \in S^{2}$ and the remainder $O\left(\frac{1}{|y|^{2}}\right)$ is uniform with respect to $x$ running through any fixed bounded domain.

We can now formulate a recipe for creating material with a desired refraction coefficient:

Recipe: To obtain material with a desired refraction coefficient $n^{2}(x)$, given a bounded domain $D$, filled with a material with the coefficient $n_{0}^{2}(x)$, one embeds into $D$ small balls $D_{m}$, centered at $x_{m}$ and of radius $a$, distributed according to (5), with $\phi(a)$ defined in (12), so that conditions (9)-(11) hold. Then one finds $h(x)$ and $N(x)$ from the equation

$$
k^{2}\left[n_{0}^{2}(x)-n^{2}(x)\right]:=p(x)=4 \pi h(x) N(x) .
$$

Equation (27) for $N(x) \geq 0$ and $h(x), \operatorname{Im} h \leq 0$, where $p(x)$ is given, has infinitely many solutions. For example, one can choose $N(x)>0$ arbitrary, and then find uniquely $h_{1}$ and $h_{2}$ using the formulas

$$
h_{1}:=\operatorname{Re} h=\frac{p_{1}}{4 \pi N(x)}, \quad h_{2}:=\operatorname{Im} h=\frac{p_{2}}{4 \pi N(x)}
$$

where $p=p_{1}+i p_{2}, p_{1}=\operatorname{Re} p, p_{2}=\operatorname{Im} p$.

By Theorem 5 the material with the embedded small particles has the desired coefficient $n^{2}(x)$ with an error that goes to zero as $a \rightarrow 0$.

We do not give proofs of Theorem 1-5. These proofs can be found in the cited papers. Our goal is to formulate clearly the recipe for creating materials with a desired $n^{2}(x)$, so that engineers and physicists can try to implement this recipe practically.

\section{A discussion of the recipe}

There are two technological problems that should be solved in order for the recipe be implemented practically.

Problem 1: How does one embed many small particles in a given material so that the desired distribution law (5),(11)-(12) is satisfied?

Problem 2: How does one prepare a small particle with the desired boundary impedance $\zeta_{m}=\frac{h\left(x_{m}\right)}{a^{\varkappa}}$ ?

The first problem, possibly, can be solved by stereolitography. The second problem one should be able to solve because the limiting cases $\zeta_{m}=0$ (hard particles) and $\zeta_{m}=\infty$ (soft particles) can be prepared, so that any intermediate value of $\zeta_{m}$ one should be able to prepare as well. The author formulates the above technological problems in the hope that engineers will get interested and solve them practically. 


\subsection{Negative refraction}

Material with negative refraction is, by definition, a material in which group velocity is directed opposite to the phase velocity, (see [1] and references therein). Group velocity is defined by the formula $\mathbf{v}_{g}=\nabla_{\mathbf{k}} \omega(\mathbf{k})$. Phase velocity $\mathbf{v}_{p}$ is directed along the wave vector $\mathbf{k}^{0}=\frac{\mathbf{k}}{|\mathbf{k}|}$. In an isotropic material $\omega=\omega(|\mathbf{k}|)$, and $\omega=\frac{c|\mathbf{k}|}{n(x, \omega)}$, so $\omega n(x, \omega)=c|\mathbf{k}|$. Differentiating this equation yields

$$
\nabla_{\mathbf{k}} \omega\left[n(x, \omega)+\omega \frac{\partial n}{\partial \omega}\right]=c \mathbf{k}^{0} .
$$

Thus,

$$
\mathbf{v}_{g}\left[n+\omega \frac{\partial n}{\partial \omega}\right]=c \mathbf{k}^{0} .
$$

Wave speed in the material with refraction coefficient $n(x, \omega)$ is $\left|\mathbf{v}_{p}\right|=\frac{c}{n(x, \omega)}$, where $c$ is the wave speed in vacuum (in $D^{\prime}$ ).

For $\mathbf{v}_{g}$ to be directed opposite to $\mathbf{k}^{0}$, that is, opposite to $\mathbf{v}_{p}$, it is necessary and sufficient that

$$
n+\omega \frac{\partial n}{\partial \omega}<0 .
$$

If the new material has $n(x, \omega)$ satisfying $(31)$, then the new material has negative refraction.

One can create material with $n(x, \omega)$ satisfying $(31)$ by choosing $h=h(x, \omega)$ properly. Namely, (27) implies

$$
n^{2}(x, \omega)=n_{0}^{2}(x)-4 \pi k^{-2} N(x) h(x, \omega) .
$$

Assuming $n_{0}^{2}>4 \pi k^{-2} N(x) h(x, \omega), \operatorname{Im} h=0$, one has (31) satisfied if

$$
\sqrt{n_{0}^{2}-4 \pi k^{-2} N(x) h(x, \omega)}<\frac{2 \pi k^{-2} N(x) \frac{\partial h(x, \omega) \omega}{\partial \omega}}{\sqrt{n_{0}^{2}-4 \pi k^{-2} N(x) h(x, \omega)}},
$$

or

$$
t(x):=\frac{n_{0}^{2}(x)}{2 \pi k^{-2} N(x)}<\omega \frac{\partial h}{\partial \omega}+2 h .
$$

This inequality has many solutions; e.g., the function $h(x, \omega)=t(x)$ solves (33).

Let us formulate the technological problem whose solving allows one to implement practically our method for creating materials with negative refraction.

Problem 3: How does one prepare a small particle with the impedance $\zeta_{m}=\frac{h\left(x_{m}, \omega\right)}{a^{\varkappa}}$, where $h(x, \omega)$ satisfies (33)?

\section{Appendix}

In the discussion after formula (32) we did not differentiate $k^{-2}$ with respect to $\omega$. If $\omega^{2} n^{2}=c^{2} k^{2}$, then (31) holds if the following inequality holds:

$$
\psi:=n+\omega \frac{\partial n}{\partial \omega}=\frac{n-\frac{2 \pi c^{2} N h^{\prime}}{\omega n^{3}}}{1-\frac{4 \pi c^{2} N h}{\omega^{2} n^{4}}}<0
$$


If $0<\frac{4 \pi c^{2} N h}{\omega^{2} n^{4}}<1$, then (34) holds if $n<\frac{2 \pi c^{2} N h^{\prime}}{\omega n^{3}}$.

This inequality holds if $n>0$ and $h^{\prime}>\frac{\omega n^{4}}{2 \pi c^{2} N}$, or, if $n<0$ and $h^{\prime}<\frac{\omega n^{4}}{2 \pi c^{2} N}$.

If $\frac{4 \pi c^{2} N h}{\omega^{2} n^{4}}>1$, then (34) holds if $n>\frac{2 \pi c^{2} N h^{\prime}}{\omega n^{3}}$.

This inequality holds if $n>0$ and $h^{\prime}<\frac{\omega n^{4}}{2 \pi c^{2} N}$, or if $n<0$ and $h^{\prime}>\frac{\omega n^{4}}{2 \pi c^{2} N}$.

\section{References}

[1] V. M. Agranovich, Yu., Gartstein, Spatial dispersion and negative refraction of light, Physics-Uspekhi, 176, N10,(2006),1051-1068.

[2] A. G. Ramm, Scattering by obstacles, D.Reidel, Dordrecht, 1986.

[3] A. G. Ramm, Inverse problems, Springer, New York, 2005.

[4] A. G. Ramm, Wave scattering by small bodies of arbitrary shapes, World Sci. Publishers, Singapore, 2005.

[5] A. G. Ramm, Equations for the self-consistent field in random medium, Phys.Lett. A, 312, N3-4, (2003), 256-261.

[6] A. G. Ramm, Scattering by many small bodies and applications to condensed matter physics, Europ. Phys. Lett., 80, (2007), 44001.

[7] A. G. Ramm, Many-body wave scattering by small bodies and applications, J. Math. Phys., 48, N10, (2007), 103511.

[8] A. G. Ramm, A recipe for making materials with negative refraction in acoustics, Phys. Lett. A, 372/13, (2008), 2319-2321.

[9] A. G. Ramm, Wave scattering by many small particles embedded in a medium, Phys. Lett. A, 372/17, (2008), 3064-3070.

[10] A. G. Ramm, Creating wave-focusing materials, LAJSS (Latin-American Journ. of Solids and Structures), 5, (2008), 119-127.

[11] A. G. Ramm, Electromagnetic wave scattering by small bodies, Phys. Lett. A, 372/23, (2008), 4298-4306.

[12] A. G. Ramm, Electromagnetic wave scattering by many conducting small particles, J. Phys A, 41, (2008), 212001.

[13] A. G. Ramm, Inverse scattering problem with data at fixed energy and fixed incident direction, Nonlinear Analysis: Theory, Methods and Applications, 69, N4, (2008), $1478-1484$. 